\title{
Structural and Functional Brain Changes in Migraine
}

Sait Ashina (D) · Enrico Bentivegna · Paolo Martelletti •

Katharina Eikermann-Haerter

Received: December 7, 2020 / Accepted: February 2, 2021 / Published online: February 16, 2021

(C) The Author(s) 2021

\section{ABSTRACT}

Migraine is a prevalent primary headache disorder and is usually considered as benign. However, structural and functional changes in the brain of individuals with migraine have been reported. High frequency of white matter abnormalities, silent infarct-like lesions, and volumetric changes in both gray and white matter in individuals with migraine compared to controls have been demonstrated. Functional magnetic resonance imaging (MRI) studies

\section{S. Ashina $(\bowtie)$}

Department of Neurology and Department of Anesthesia, Critical Care and Pain Medicine, BIDMC Comprehensive Headache Center, Beth Israel Deaconess Medical Center, Harvard Medical School, Boston, MA, USA

e-mail: sashina@bidmc.harvard.edu

\section{S. Ashina}

Department of Clinical Medicine, Faculty of Health Sciences, University of Copenhagen, Copenhagen, Denmark

E. Bentivegna · P. Martelletti

Internal Medicine and Emergency Medicine, Sant'Andrea Hospital, Sapienza University, Rome, Italy

\section{P. Martelletti}

Department of Clinical and Molecular Medicine, Sapienza University, Rome, Italy

K. Eikermann-Haerter

Department of Radiology, Massachusetts General

Hospital, Harvard Medical School, Boston, MA, USA found altered connectivity in both the interictal and ictal phase of migraine. MR spectroscopy and positron emission tomography studies suggest abnormal energy metabolism and mitochondrial dysfunction, as well as other metabolic changes in individuals with migraine. In this review, we provide a brief overview of neuroimaging studies that have helped us to characterize some of these changes and discuss their limitations, including small sample sizes and poorly defined control groups. A better understanding of alterations in the brains of patients with migraine could help not only in the diagnosis but may potentially lead to the optimization of a targeted anti-migraine therapy.

Keywords: Brain; Functional; Migraine; MRI; Neuroimaging; PET; Spectroscopy; Structural

\section{Key Summary Points}

Migraine is a highly prevalent primary headache disorder, usually considered as benign.

However, migraine is associated with an increased vulnerability to ischemia and stroke, particularly in women with migraine with aura and younger than age 45. 
Recent neuroimaging studies characterize structural and functional changes in the brains of patients with migraine that include:

- High frequency of white matter abnormalities,

- Silent infarct-like lesions,

- Volumetric changes in both gray and white matter,

- Altered functional connectivity in both the interictal and ictal phase,

- Abnormal energy metabolism, mitochondrial dysfunction, and additional metabolic changes in the brain.

A better understanding of alterations in the brains of patients with migraine could help not only in the diagnosis but may potentially help develop a targeted anti-migraine therapy.

\section{DIGITAL FEATURES}

This article is published with digital features, including a summary slide, to facilitate understanding of the article. To view digital features for this article go to https://doi.org/10.6084/ m9.figshare.13674793.

\section{INTRODUCTION}

Migraine is a highly prevalent primary headache with estimated global 1-year prevalence of $15 \%$ of the general population [1-3]. Considered a chronic neurovascular disorder with episodic manifestations, migraine has enormous socioeconomic impact on the individual and society $[3,4]$. The pathophysiology of migraine is complex and not fully clarified $[3,5]$. Cortical spreading depolarization (CSD) and abnormal brain stem activity have been shown to be involved in the pathophysiology of migraine with aura [3, 6-8]. The pain in migraine most likely originates in the nociceptive sensory fibers transmitting signals from intracranial and extracranial blood vessels, and other cranial structures such as dura mater, skin, muscles, and periosteum $[3,9]$. Peripheral and central sensitization of trigeminovascular nociceptive pathways may develop during migraine attacks $[3,10,11]$. Alterations of the trigeminovascular pathways and dysregulation of vasodilator peptides involved in this network such as calcitonin gene-related peptide (CGRP) have been the subject of recent studies on pathophysiology and therapies of this disease [12, 13]. Genetic predisposition and its repercussions on brain networks are other known elements underlying the pathophysiology of migraine that have made it possible to better understand the underlying functional alterations [14-17].

Migraine is considered a benign disease, but long-term effects of migraine have been debated. Recent evidence from neuroimaging studies including interictal magnetic resonance imaging (MRI) shows that migraine pathology may extend beyond migraine pain and can be associated with structural and functional brain changes [18-21]. Migraine changes the brain in ictal and interictal states, resulting in altered morphology of cerebral, cerebellar, and brainstem structures, as well as altered neuronal networks and function. These findings from human studies are supported by evidence from animal studies [22, 23]. For instance, in vivo multiphoton microscopy demonstrated increased intraneuronal $\mathrm{Ca}^{2+}$ concentrations $\left[\mathrm{Ca}^{2+}\right]_{\mathrm{i}}$ both at resting state and during CSD in a migraine mouse model carrying human mutations for the rare migraine subtype familial hemiplegic migraine type 1 (FHM1). This finding was associated with stronger synaptic connections in FHM1 mutants, as evidenced by larger axonal boutons and a higher percentage of highly excitable mushroom-type dendritic spines, when compared to wild-type littermates [22]. These changes may contribute to the persistently hyperexcitable state in FHM1, and possibly other types of migraine. In the current review, we describe findings from neuroimaging studies on structural and functional changes in the brain of individuals with migraine.

This article is based on previously conducted studies and does not contain any new studies with human participants or animals performed by any of the authors 


\section{STRUCTURAL CHANGES}

There are several types of structural brain abnormalities that have been reported in individuals with migraine. Neuroimaging studies often reveal white matter hyperintensities or white matter abnormalities (WMAs), silent infarct-like lesions (ILLs), ischemic lesions (stroke), as well as volumetric changes in gray (GM) and white matter (WM) in migraine sufferers $[18,19]$.

\section{White Matter Abnormalities}

WMAs are usually small punctate hyperintense lesions localized to the deep, subcortical, periventricular, and infratentorial structures, with no associated mass effect [18, 24-27]. WMAs are typically seen on T2 and fluid-attenuated inversion recovery (FLAIR) MR images, and assumed to be composed of gliosis, demyelination, and loss of axons, possibly resulting from microvascular damage [28]. WMAs in migraine have been extensively studied in clinic-based and population-based studies, and two meta-analyses of these studies have been conducted [18, 29-35]. The clinic-based studies did not include migraine-free control groups and may therefore not be generalizable because of selection bias [18]. Association between WMA and migraine has mainly been reported in cross-sectional and only in few longitudinal population-based studies. Individuals with migraine are up to 2-4 times more likely to have WMAs detected on conventional MRIs of the brain compared to controls [18, 30, 31, 34, 36]. Moreover, several studies indicate that migraine with aura, particularly in women, is associated with an increased risk of WMAs $[18,29]$. In the systematic review conducted by Bashir et al. [18], the relative frequency of WMAs in individuals with migraine ranged from $4 \%$ to $59 \%$. The risk of WMA in migraine was shown to be independent of cardiovascular risk factors in previous studies $[24,26,27]$. However, it must be noted that the results of the previous studies are variable, inconsistent, and at times conflicting, possibly as a result of methodological differences
$[33,36]$. In the Cerebral Abnormalities in Migraine, an Epidemiological Risk Analysis 1 (CAMERA-1) study, the prevalence of WMAs was studied in individuals with migraines and gender-matched controls [26]. The prevalence of deep WMAs was higher in individuals with migraine when compared to controls, independent of the presence of aura and increased with attack frequency in women. Additional analysis [27] of the same population revealed that hyperintense lesions were found in the infratentorial structures, cerebellum and brain stem, in individuals with migraine. ILLs were associated with supratentorial WMAs but not with migraine subtype, migraine attack frequency, or cerebellar infarcts. The Epidemiology of Vascular Ageing-MRI (EVA-MRI) study [25] also confirmed the association of migraine with WMAs. In this study, the association with deep WMAs was found to be stronger for migraine with aura than without aura. Interestingly, no association was found between overall headache status, WMAs, and cognitive impairment in the EVA-MRI study [25].

CAMERA-2 [24] is a prospective 9-year follow-up study that aimed to assess the association between migraine attack frequency and progression/increase in number and volume of WMAs in the same study population. This study showed that women with migraine, migraine without aura in particular, had a higher incidence of deep WMA progression compared to controls. Notably, progression of WMAs in individuals with migraine was not associated with migraine attack frequency, duration, severity, or anti-migraine treatments. There was no association between WMA volume and cognitive dysfunction at follow-up. This finding is in agreement with a prospective study by Rist et al. [37], which demonstrated no link between cognitive changes and WMAs in individuals with migraine. Moreover, no significant associations of migraine with progression of infratentorial hyperintensities was reported in the CAMERA-2 study [24]. Another longitudinal study [38], which did not have a non-migraine control group, suggested WMA progression in individuals with migraine with aura and correlation with headache attack frequency. However, in a more recent prospective study by 
Hamedani et al. [34], it was demonstrated that there was no difference in WMA progression between those with and without migraine. The observed stable associations between migraine and WMAs in older age suggested that white matter changes were occurring earlier in life [34]. The shortcomings of this study were limited white matter analyses, retrospective headache case definitions, lack of full use of the International Headache Society (ICDH) diagnostic criteria, and exclusion of individuals with history of severe headache.

The pathophysiological mechanisms leading to the development of WMAs in migraine and histopathological correlates of WMA are not fully understood. Ischemic microvascular disturbances with subsequent regional hypoperfusion of the brain [39], brain injury due to activated metalloproteinases during CSD [40], microembolism, coagulation activation, and endothelial dysfunction [41, 42] have been suggested as possible underlying mechanisms.

\section{Silent Infarct-Like Lesions}

Silent ILLs are MRI or computed tomography (CT) signal abnormalities defined as non-mass parenchymal defects, having a size of at least $3 \mathrm{~mm}$, while being isointense to cerebrospinal fluid signal on all sequences [43]. ILLs can be surrounded by a hyperintense rim on FLAIR and proton density MR images [18]. Dilated perivascular spaces may be difficult to differentiate from caveated ILLs. The distinction is mainly based on the diameter of the defect, with those greater than $3 \mathrm{~mm}$ of diameter most likely being ILLs [43]. The mechanisms leading to ILLs are not fully understood but hypoperfusion or microemboli may be possible causative factors [18]. It has also been suggested that ILLs may be a manifestation of small-vessel disease related to hypertension [44].

The association between migraine and silent ILLs has been reported in both clinic-based and population-based studies [18, 32, 45]. A high frequency of ILLs in the posterior circulation distribution areas, mostly in the cerebellum, in individuals with migraine with aura compared to those without aura or controls has been reported in two cross-sectional studies [26, 46]. In one cross-sectional study, Kurth et al. [25] reported an increased risk of ILLs mostly in deep gray matter, subcortex and the basal ganglia, in individuals with migraine with aura. Interestingly, ILLs were not reported to be associated with supratentorial WMAs [46]. In the Northern Manhattan Study (NOMAS) [33], migraine was associated with a twofold likelihood of ILLs after adjusting for several variables including body mass index, cardiovascular risk factors, smoking, and demographics [adjusted odds ratio (OR) 2.1; 95\% confidence interval (CI) 1.0-4.2]. The association was stronger for migraine without aura, but the results should be interpreted with caution given the restrictive definition of aura in the study. In a small-sized cohort study of 100 women [45] with chronic migraine with or without aura, posterior circulation ILLs were identified in $6 \%$ of the patients in comparison to no infarct-like lesions in 15 controls with episodic migraine. In contrast to the aforementioned studies, Geist et al. [36] did not demonstrate differences in frequency of ILLs between individuals with migraine with aura and controls. However, this study has several methodological limitations including non-participation, and questionable generalizability of twin studies.

A prospective study in individuals with migraine (CAMERA-2) did not demonstrate a statistically significant increased risk of progression of the previously found ILLs [24]. This negative result has been suggested to be due to lack of power [18]. Additionally, CAMERA-2 showed no association between cognitive function and ILLs. In a prospective population study from Iceland [47], Scher et al. showed that women with midlife migraine with aura had a twofold increased risk of late-life cerebellar ILLs at follow-up 26 years later. ILLs were independent of late-life cardiovascular risks and history of coronary artery disease, transient ischemic attacks, or stroke.

The causative relationship between migraine and ILLs is not clear. It is also unknown whether silent ILLs predispose to the development of clinical stroke $[18,32]$. It has been shown that individuals with migraine and with ILLs have a less favorable cardiovascular risk profile than 
those without ILLs and a higher prevalence of clinical stroke [24]. Clinical significance of ILLs in migraine is not fully clarified but may support subclinical cerebellar impairment reported in patients with migraine [48].

\section{Volumetric Changes in Gray and White Matter}

Volumetric changes can be detected by using voxel-based morphometry (VBM) or diffusion tensor imaging (DTI). Voxel-based morphometry is a computational approach that measures differences in local concentrations of brain tissue, through a voxel-wise comparison of multiple brain images with a template. DTI allows for visualization of the orientation and anisotropy (a measure reflecting fiber density, axonal diameter, and myelination) of white and gray matter by measuring the diffusion of water. Volumetric changes are visualized and described as decreased or increased gray matter or white mater volumes [49-53]. Pathophysiology of volumetric changes likely involves repeated episodes of ischemia caused by cerebral blood flow abnormalities during both the ictal and in the interictal phases of migraine [18].

There are several studies that used VBM and DTI to assess volumetric changes in gray and white matter regions in individuals with migraine compared to controls. Volume loss was demonstrated in the bilateral insula, frontal/prefrontal, temporal, parietal, and occipital cortices, the anterior cingulate cortex (ACC), basal ganglia, and the cerebellum in patients with migraine compared to controls [49, 50, 54-59]. Increased gray matter density in the periaqueductal gray (PAG) and the dorsolateral pons was reported in patients with migraine with aura [54]. Furthermore, migraine attack frequency and migraine disease duration were correlated with gray matter reduction in individuals with migraine in the frontal, temporal, and parietal lobes, the limbic system, the ACC, the brainstem, and cerebellum [49-51, 54, 55, 57, 60]. In one study [50], patients with chronic migraine had volume loss in the ACC and in several other areas, compared to patients with episodic migraine, also suggesting an association between attack frequency and the degree of gray matter reduction. When compared to controls, individuals with chronic migraine were shown to have smaller volumes of the cerebellum and brainstem [35]. Volumetric changes may well indicate brain remodeling in migraine. Clinical implications of volumetric changes are unknown and may reflect a consequence of frequent migraine attacks, but additional studies are needed to confirm this assumption.

\section{FUNCTIONAL CHANGES}

Functional MRI (fMRI) is an often-used imaging method to study neuronal network connectivity [21]. fMRI utilizes blood oxygen level dependent (BOLD) recordings of the brain as an indirect measure of neuronal activity that reflects changes in regional cerebral blood flow, volume, and oxygenation. Each voxel in the obtained image of the brain represents a signal with a specific frequency. If there is a high degree of synchronization of signal frequencies between two different voxels, then these are functionally connected or even constitute a functional network. There are two types of fMRI studies that can be performed: resting state and task-based [61]. Resting-state fMRI studies investigate synchronicity of spontaneous fluctuations in the BOLD signal as a measure of neuronal connectivity in the absence of a specific stimulus, while the subject is lying in the scanner with eyes closed but not sleeping [62]. During task-based fMRI, the subject performs a task while being scanned.

Resting-state fMRI studies demonstrate functional changes in certain areas of the brain in patients with migraine, including in the diencephalon and brainstem nuclei during the interictal and headache phases [20, 63-72].

During the interictal phase, alterations have been recognized in more than 20 functional connectivity networks in patients with migraine compared to those without migraine, including the default mode network, salience network, frontoparietal network, executive network, and sensorimotor network [73]. There is also increased activation of the visual cortex 
$[74,75]$, primary sensorimotor cortex [76], superior-anterior middle temporal complex [77], and perigenual anterior cingulate cortex [78-80], with an increased connection between nociceptive areas and the periaqueductal gray. Interestingly, the extent of abnormalities positively correlates with markers of migraine severity such as headache frequency and number of years with migraine [81]. Some studies suggest differences in interictal alterations of resting state functional connectivity between individuals with migraine with and without aura, involving the visual cortex as well as widespread regions involved in visual processing (including the middle frontal areas, the insula, the anterior cingulate, the superior parietal lobule, and the cerebellum). Results indicate that those with aura compared to those without aura have weaker functional connectivity between the anterior insula and the V3A in the visual cortex, which was inversely related to migraine severity [82]. In contrast, Tedeschi et al. found stronger connectivity within the visual network centering around the lingual gyrus, an extrastriate region important for visual-spatial processing, while Hougaard et al. did not identify any differences in functional connectivity between migraineurs and non-migraineurs $[83,84]$.

During the headache phase, there is evidence for altered connectivity involving the salience network, the somatosensory network, the default mode network, the pons, and the thalamus [21]. There is increased activity within brainstem nuclei, the cerebellum, putamen, temporal lobes, prefrontal cortex, precentral and postcentral gyrus, cingulate, thalamus, and insula [68-72]. Coppola et al. [85] utilized whole-brain independent component analysis during naturally occurring (not medication-induced) migraine attacks and showed decreased functional connectivity between the executive and dorsoventral attention network, when compared to healthy controls, which correlated with attack frequency. In addition, the authors found evidence for functional decoupling of the thalamocortical control network. Again, weaker executive network connectivity related to higher monthly headache frequency was noted in patients with migraine. In a follow-up study, using the same study population, the authors found stronger connectivity between the medial prefrontal cortex and the posterior cingulate cortex, as well as stronger connectivity between the medial prefrontal cortex and the insula, suggestive of altered emotional processing of pain [86]. In chronic migraine, there is evidence for reduced connectivity of the default mode network with the executive control network, and increased connectivity with the dorsal attention system, with the latter showing decreased connectivity with the executive control network. These findings correlate with headache severity [87]. Another study showed decreased overall functional connectivity of the three major intrinsic brain networks (default mode, salience, central executive networks) in women with chronic migraine [88].

In summary, fMRI studies show altered connectivity in both interictal and ictal migraine, which could help improve our understanding of underlying disease mechanisms. However, findings are equivocal and partially contradicting, while so far no reproducible biomarkers of migraine could be identified. Differences in imaging protocols as well as the fact that migraine is a heterogeneous disorder with different disease duration, attack frequency, comorbidity, effect of treatment, presence or absence of aura might have caused variations in results between studies.

\section{METABOLIC CHANGES}

\section{MRS Studies}

Magnetic resonance spectroscopy (MRS) is a non-invasive imaging method, which provides in vivo molecular information related to the metabolism and function of brain structures $[89,90]$. For example, phosphorus $\left({ }^{31} \mathrm{P}\right)$-MRS can measure concentrations of chemicals containing phosphorus nuclei that include inorganic phosphate, phosphocreatine (PCr), creatine, adenosine diphosphate (ADP), and adenosine triphosphate (ATP). Hydrogen $\left({ }^{1} \mathrm{H}\right)$ MRS measures signals from proton nuclei in water and allows us to measure PCr, creatine, 
choline, $\mathrm{N}$-acetylaspartate (NAA), myo-inositol, glutamate, GABA, aspartate, and lactate.

Overall, MRS studies suggest that there is abnormal energy metabolism and mitochondrial dysfunction in patients with migraine, which may decrease the threshold for initiating migraine attacks $[89,91]$. Decreased PCr and increased inorganic phosphate, or decreased $\mathrm{PCr} /$ inorganic phosphate ratio, were reported in several cortical areas including the occipital cortex in patients with migraine with and without aura both during attacks and in the interictal state, when using ${ }^{31}$ P-MRS [92-95]. In addition, decreased phosphorylation potential, an index of available free energy in the cell, was shown in patients with and without aura, indicating less freely available energy [92, 95-97]. Another important finding was decreased magnesium in cortical areas in individuals with migraine with and without aura, which also suggests mitochondrial dysfunction and decreased availability of energy in neurons [92, 93, 98-100].

The ${ }^{1} \mathrm{H}$-MRS studies demonstrate changes in the levels of glutamate, a neurotransmitter possibly involved in cortical hyperexcitability. Increased glutamate levels interictally were detected in the visual and anterior paracingulate cortex, and in the visual cortex during visual stimulation in migraine with aura [101-103]. These findings, together with reports of decreased interictal GABA levels in the occipital lobe of patients with migraine with and without aura, suggest a predominance of excitatory over inhibitory connections in migraineurs [101, 104]. Decreased NAA levels indicating neuronal loss were found in the occipital cortex and thalamus in subjects with migraine without aura [105-107]. Limitations of MRS studies have been previously described $[89$, 108]. These include identification and quantification of metabolites. Results from MRS studies in migraine revealed the use of different methodologies, mainly performed in the interictal state. Therefore, the results of these studies may not be comparable, with only few study findings being reproducible.

\section{PET Studies}

A brain positron emission tomography (PET) scan is an imaging test which uses radiotracers that emit positrons, which then undergo radioactive decay and collide with electrons to produce two photons [109]. PET scan can detect these photons and produce an image of spatial density, showing tracer uptake/binding changes.

Using the $5-\mathrm{HT}_{1 \mathrm{~B}}$ receptor-specific radioligand $\left[{ }^{11} \mathrm{C}\right] \mathrm{AZ10419369}$, Deen et al. [110] demonstrated lower $5-\mathrm{HT}_{1 \mathrm{~B}}$ binding in patients with migraine in pain-processing regions of the brain, including the anterior cingulate cortex, sensorimotor cortex, the insula, and amygdala, suggestive of a decreased density of the $5-\mathrm{HT}_{1 \mathrm{~B}}$ receptor in patients with migraine.

A recent study [111] using integrated PET/ MRI brain scans with $\left[{ }^{11} \mathrm{C}\right] \mathrm{PBR} 18$, a radioligand that binds to the $18 \mathrm{kDa}$ translocator protein (TSPO), demonstrated that patients with migraine interictally and at least 2 weeks following the headache attack had elevated standardized uptake value ratio (SUVR) in their visual cortex as well as the thalamus, primary/ secondary somatosensory, and insular cortices. These findings suggest glial activation in subjects with migraine with aura and neuroimmune activation/neuroinflammation in brain regions involved in migraine pathophysiology including generation of CSD as well as nociception [112]. Interestingly, strong persistent extra-axial inflammatory signal was found in the meninges and calvarial bone overlying the occipital lobe in patients with migraine during and after visual auras, implicating newly discovered bridging vessels that facilitate bidirectional crosstalk between brain and skull marrow [113]. Inflammatory cells in the calvarium may serve as a local repository and as a potential candidate to trigger subsequent attacks, possibly promoting migraine chronification.

PET studies have also made it possible to study different metabolic activities in the various phases of migraine. During the aura, for example, decreased perfusion of the parietal and temporal lobes has been observed $[114,115]$. During the migraine attack instead, PET studies identified greater blood flow in the 
brainstem nuclei and cerebellum [68, 71, 116], and decreased metabolism of the thalamus, orbitofrontal cortex, anterior cingulate cortex, and ventral striatum [117]. In addition, it was demonstrated that the degree of neuroinflammation correlated with the frequency of migraine attacks [111]. Limitations of this study were the small sample size and lack of kinetic modeling with radiometabolite-corrected arterial input function for better quantification of TSPO binding.

\section{PERSPECTIVES AND CONCLUSIONS}

There is increasing evidence for migraine as a potential risk factor for structural and functional changes in the brain. Individuals with migraine seem to have more WMAs, ILLs, and volumetric changes in both gray and white matter when compared to controls. Epidemiological studies suggest an association between migraine and stroke. Moreover, fMRI studies have shown that individuals with migraine may have atypical neuronal networks and function. However, the majority of the conducted studies have flaws and methodological limitations. These include small sample sizes, often not whole-brain but selective brain region analyses, variation in methods of data collection, differences in timing of data collection with respect to migraine attack, and no account of the use of acute or preventive treatments. In addition, there is a shortage of replication studies. Identifying areas involved in the pathophysiology of migraine in functional imaging methods has proven to be difficult, and the same is true for defining diagnostic standards regarding brain functional alterations in migraine [21]. Furthermore, more studies are needed to understand whether these functional abnormalities are part of the causes of migraines and/or if they are rather the consequences of repeated attacks. As a result of the cross-sectional nature of the vast majority of studies, we are still unable to ascertain whether the structural and functional changes in the brain of the individual with migraine are secondary to migraine. To answer this question, prospective studies are warranted. Longitudinal studies are also needed to evaluate how a change in clinical phenotype of headache such as transformation of episodic to chronic migraine can affect brain imaging findings. A better understanding of changes in structure and function of brain in individuals with migraine could help not only in the diagnosis but also in the therapeutic choice [64]. It has been suggested that the evaluation of changes in functional studies could potentially guide the optimization of therapy.

\section{ACKNOWLEDGEMENTS}

Funding. No funding or sponsorship was received for this study or publication of this article.

Authorship. All named authors meet the International Committee of Medical Journal Editors (ICMJE) criteria for authorship for this article, take responsibility for the integrity of the work as a whole, and have given their approval for this version to be published.

Authorship Contributions. Paolo Martelletti and Katharina Eikermann-Haerter contributed equally to the manuscript.

Disclosures. Sait Ashina received honoraria for consulting from Allergan, Amgen, Biohaven, Eli Lilly, Impel NeuroPharma, Novartis, Satsuma, Supernus, Theranica, Percept. He is an associate editor for Neurology Reviews and serves on Advisory Board for Journal of Headache and Pain. Enrico Bentivegna has nothing to disclose. Paolo Martelletti received Research Grants to his University Department from Allergan and Novartis, fees for webinars from Allergan, Lilly, Novartis. Fees and Royalties from SpringerNature. He is Editor-on-Chief of The Journal of Headache and Pain, Editor-in-Chief of SN Comprehensive Clinical Medicine and Expert of European Medicine Agency. Katharina EikermannHaerter received a Research Grant from the Massachusetts General Hospital (Schlaeger Award). She is Associated Editor of Frontiers in Neurology. 
Compliance with Ethics Guidelines. This article is based on previously conducted studies and does not contain any new studies with human participants or animals performed by any of the authors.

Open Access. This article is licensed under a Creative Commons Attribution-NonCommercial 4.0 International License, which permits any non-commercial use, sharing, adaptation, distribution and reproduction in any medium or format, as long as you give appropriate credit to the original author(s) and the source, provide a link to the Creative Commons licence, and indicate if changes were made. The images or other third party material in this article are included in the article's Creative Commons licence, unless indicated otherwise in a credit line to the material. If material is not included in the article's Creative Commons licence and your intended use is not permitted by statutory regulation or exceeds the permitted use, you will need to obtain permission directly from the copyright holder. To view a copy of this licence, visit http://creativecommons.org/licenses/by$\mathrm{nc} / 4.0 /$.

\section{REFERENCES}

1. Lipton RB, Bigal ME, Diamond M, Freitag F, Reed ML, Stewart WF. Migraine prevalence, disease burden, and the need for preventive therapy. Neurology. 2007;68:343-9.

2. Steiner TJ, Stovner LJ, Katsarava Z, et al. The impact of headache in Europe: principal results of the Eurolight project. J Headache Pain. 2014;15:31-31.

3. Ashina M. Migraine. N Engl J Med. 2020;383: 1866-76.

4. GBD 2016 Headache Collaborators. Global, regional, and national burden of migraine and tensiontype headache, 1990-2016: a systematic analysis for the Global Burden of Disease Study 2016. Lancet Neurol. 2018;17:954-976.

5. Ashina S, Bendtsen L, Ashina M. Pathophysiology of migraine and tension-type headache. Tech Reg Anesthesia Pain Manag. 2012;16:14-8.
6. Noseda R, Burstein R. Migraine pathophysiology: anatomy of the trigeminovascular pathway and associated neurological symptoms, CSD, sensitization and modulation of pain. Pain. 2013. https:// doi.org/10.1016/j.pain.2013.1007.1021.

7. Goadsby PJ, Holland PR, Martins-Oliveira M, Hoffmann J, Schankin C, Akerman S. Pathophysiology of migraine: a disorder of sensory processing. Physiol Rev. 2017;97:553-622.

8. Harriott AM, Takizawa T, Chung DY, Chen SP. Spreading depression as a preclinical model of migraine. J Headache Pain. 2019;20:45.

9. Olesen J, Burstein R, Ashina M, Tfelt-Hansen P. Origin of pain in migraine: evidence for peripheral sensitisation. Lancet Neurol. 2009;8:679-90.

10. Edvinsson JCA, Viganò A, Alekseeva A, et al. The fifth cranial nerve in headaches. J Headache Pain. 2020;21:65.

11. Russo A, Tessitore A, Silvestro M, et al. Advanced visual network and cerebellar hyperresponsiveness to trigeminal nociception in migraine with aura. J Headache Pain. 2019;20:46.

12. De Matteis E, Guglielmetti M, Ornello R, Spuntarelli V, Martelletti P, Sacco S. Targeting CGRP for migraine treatment: mechanisms, antibodies, small molecules, perspectives. Expert Rev Neurother. 2020;20:627-41.

13. Favoni V, Giani L, Al-Hassany L, et al. CGRP and migraine from a cardiovascular point of view: what do we expect from blocking CGRP? J Headache Pain. 2019;20:27-27.

14. de Boer I, Terwindt GM, van den Maagdenberg A. Genetics of migraine aura: an update. J Headache Pain. 2020;21:64.

15. Pietrobon D, Brennan KC. Genetic mouse models of migraine. J Headache Pain. 2019;20:79.

16. Sutherland HG, Albury CL, Griffiths LR. Advances in genetics of migraine. J Headache Pain. 2019;20: 72.

17. van den Maagdenberg A, Nyholt DR, Anttila V. Novel hypotheses emerging from GWAS in migraine? J Headache Pain. 2019;20:5.

18. Bashir A, Lipton RB, Ashina S, Ashina M. Migraine and structural changes in the brain: a systematic review and meta-analysis. Neurology. 2013;81: 1260-8.

19. Chen S-P, Eikermann-Haerter K. How imaging can help us better understand the migraine-stroke 
connection. Headache J Head Face Pain. 2020;60: 217-28.

20. Sprenger T, Borsook D. Migraine changes the brain: neuroimaging makes its mark. Curr Opin Neurol. 2012;25:252-62.

21. Skorobogatykh K, van Hoogstraten WS, Degan D, et al. Functional connectivity studies in migraine: what have we learned? J Headache Pain. 2019;20: 108-108.

22. Eikermann-Haerter K, Arbel-Ornath M, Yalcin N, et al. Abnormal synaptic $\mathrm{Ca}^{2+}$ homeostasis and morphology in cortical neurons of familial hemiplegic migraine type 1 mutant mice. Ann Neurol. 2015;78:193-210.

23. Theriot JJ, Toga AW, Prakash N, Ju YS, Brennan KC. Cortical sensory plasticity in a model of migraine with aura. J Neurosci. 2012;32:15252.

24. Palm-Meinders IH, Koppen H, Terwindt GM, et al. Structural brain changes in migraine. JAMA. 2012;308:1889-97.

25. Kurth T, Mohamed S, Maillard P, et al. Headache, migraine, and structural brain lesions and function: population based epidemiology of vascular ageingMRI study. BMJ. 2011;342:c7357.

26. Kruit MC, van Buchem MA, Hofman PA, et al. Migraine as a risk factor for subclinical brain lesions. JAMA. 2004;291:427-34.

27. Kruit MC, Launer LJ, Ferrari MD, van Buchem MA. Brain stem and cerebellar hyperintense lesions in migraine. Stroke. 2006;37:1109-12.

28. Porter A, Gladstone JP, Dodick DW. Migraine and white matter hyperintensities. Curr Pain Headache Rep. 2005;9:289-93.

29. Russo A, Silvestro M, Tessitore A, Tedeschi G. Recent insights in migraine with aura: a narrative review of advanced neuroimaging. Headache J Head Face Pain. 2019;59:637-49.

30. Swartz RH, Kern RZ. Migraine is associated with magnetic resonance imaging white matter abnormalities: a meta-analysis. Arch Neurol. 2004;61: 1366-8.

31. Zhang Q, Datta R, Detre JA, Cucchiara B. White matter lesion burden in migraine with aura may be associated with reduced cerebral blood flow. Cephalalgia. 2017;37:517-24.

32. Hougaard A, Amin FM, Ashina M. Migraine and structural abnormalities in the brain. Curr Opin Neurol. 2014;27:309-14.
33. Monteith T, Gardener H, Rundek T, et al. Migraine, white matter hyperintensities, and subclinical brain infarction in a diverse community: the northern Manhattan study. Stroke. 2014;45:1830-2.

34. Hamedani AG, Rose KM, Peterlin BL, et al. Migraine and white matter hyperintensities: the ARIC MRI study. Neurology. 2013;81:1308-13.

35. Bilgiç B, Kocaman G, Arslan AB, et al. Volumetric differences suggest involvement of cerebellum and brainstem in chronic migraine. Cephalalgia. 2016;36:301-8.

36. Gaist D, Garde E, Blaabjerg M, et al. Migraine with aura and risk of silent brain infarcts and white matter hyperintensities: an MRI study. Brain. 2016;139:2015-23.

37. Rist PM, Dufouil C, Glymour MM, Tzourio C, Kurth T. Migraine and cognitive decline in the population-based EVA study. Cephalalgia Int J Headache. 2011;31:1291-300.

38. Dinia L, Bonzano L, Albano B, et al. White matter lesions progression in migraine with aura: a clinical and MRI longitudinal study. J Neuroimaging. 2013;23:47-52.

39. Colombo B, DallaLibera D, Comi G. Brain white matter lesions in migraine: what's the meaning? NeurolSci. 2011;32(Suppl 1):S37-40.

40. Gursoy-Ozdemir Y, Qiu J, Matsuoka N, et al. Cortical spreading depression activates and upregulates MMP-9. J Clin Investig. 2004;113:1447-55.

41. Dalkara T, Nozari A, Moskowitz MA. Migraine aura pathophysiology: the role of blood vessels and microembolisation. Lancet Neurol. 2010;9:309-17.

42. Yemisci M, Eikermann-Haerter K. Aura and stroke: relationship and what we have learnt from preclinical models. J Headache Pain. 2019;20:63.

43. Zhu YC, Dufouil C, Tzourio C, Chabriat H. Silent brain infarcts: a review of MRI diagnostic criteria. Stroke. 2011;42:1140-5.

44. Vermeer SE, Longstreth WT Jr, Koudstaal PJ. Silent brain infarcts: a systematic review. Lancet Neurol. 2007;6:611-9.

45. Santamarta E, Meilán A, Saiz A, et al. Chronic migraine does not increase posterior circulation territory (PCT) infarct-like lesions. J NeurolSci. 2014;336:180-3.

46. Kruit MC, Launer LJ, Ferrari MD, van Buchem MA. Infarcts in the posterior circulation territory in migraine. The population-based MRI CAMERA study. Brain. 2005;128:2068-77. 
47. Scher AI, Gudmundsson LS, Sigurdsson S, et al. Migraine headache in middle age and late-life brain infarcts. JAMA. 2009;301:2563-70.

48. Sándor PS, Mascia A, Seidel L, De Pasqua V, Schoenen J. Subclinical cerebellar impairment in the common types of migraine: a three-dimensional analysis of reaching movements. Ann Neurol. 2001;49:668-72.

49. Kim JH, Suh SI, Seol HY, et al. Regional grey matter changes in patients with migraine: a voxel-based morphometry study. Cephalalgia. 2008;28: 598-604.

50. Valfrè W, Rainero I, Bergui M, Pinessi L. Voxelbased morphometry reveals gray matter abnormalities in migraine. Headache. 2008;48:109-17.

51. Qin Z, He X-W, Zhang J, et al. Structural changes of cerebellum and brainstem in migraine without aura. J Headache Pain. 2019;20:93.

52. Palm-Meinders IH, Arkink EB, Koppen $\mathrm{H}$, et al. Volumetric brain changes in migraineurs from the general population. Neurology. 2017;89:2066-74.

53. Schwedt TJ, Dodick DW. Advanced neuroimaging of migraine. Lancet Neurol. 2009;8:560-8.

54. Rocca MA, Ceccarelli A, Falini A, et al. Brain gray matter changes in migraine patients with T2-visible lesions: a 3-T MRI study. Stroke. 2006;37:1765-70.

55. Jin C, Yuan K, Zhao L, et al. Structural and functional abnormalities in migraine patients without aura. NMR Biomed. 2013;26:58-64.

56. Schmitz N, Arkink EB, Mulder M, et al. Frontal lobe structure and executive function in migraine patients. NeurosciLett. 2008;440:92-6.

57. Schmitz N, Admiraal-Behloul F, Arkink EB, et al. Attack frequency and disease duration as indicators for brain damage in migraine. Headache. 2008;48: 1044-55.

58. Schmidt-Wilcke T, Gänssbauer S, Neuner T, Bogdahn U, May A. Subtle grey matter changes between migraine patients and healthy controls. Cephalalgia. 2008;28:1-4.

59. Bonanno L, Lo Buono V, De Salvo S, et al. Brain morphologic abnormalities in migraine patients: an observational study. J Headache Pain. 2020;21:39.

60. Chen X-Y, Chen Z-Y, Dong Z, Liu M-Q, Yu S-Y. Regional volume changes of the brain in migraine chronification. Neural Regen Res. 2020;15:1701-8.
61. Soares JM, Magalhães R, Moreira PS, et al. A Hitchhiker's guide to functional magnetic resonance imaging. Front Neurosci. 2016;10:515.

62. Lv H, Wang Z, Tong E, et al. Resting-state functional MRI: everything that nonexperts have always wanted to know. Am J Neuroradiol. 2018;39:1390-9.

63. Huang X, Zhang D, Chen Y, et al. Altered functional connectivity of the red nucleus and substantianigra in migraine without aura. J Headache Pain. 2019;20: 104.

64. Lakhan SE, Avramut M, Tepper SJ. Structural and functional neuroimaging in migraine: insights from 3 decades of research. Headache. 2013;53:46-66.

65. Long T, He W, Pan Q, et al. Microglia P2X4R-BDNF signalling contributes to central sensitization in a recurrent nitroglycerin-induced chronic migraine model. J Headache Pain. 2020;21:4.

66. Andreou AP, Edvinsson L. Mechanisms of migraine as a chronic evolutive condition. J Headache Pain. 2019;20:117.

67. Torres-Ferrús M, Ursitti F, Alpuente A, et al. From transformation to chronification of migraine: pathophysiological and clinical aspects. J Headache Pain. 2020;21:42.

68. Weiller C, May A, Limmroth V, et al. Brain stem activation in spontaneous human migraine attacks. Nat Med. 1995;1:658-60.

69. Cao Y, Aurora SK, Nagesh V, Patel SC, Welch KM. Functional MRI-BOLD of brainstem structures during visually triggered migraine. Neurology. 2002;59: 72-8.

70. Bahra A, Matharu MS, Buchel C, Frackowiak RS, Goadsby PJ. Brainstem activation specific to migraine headache. Lancet. 2001;357:1016-7.

71. Afridi SK, Giffin NJ, Kaube H, et al. A positron emission tomographic study in spontaneous migraine. Arch Neurol. 2005;62:1270-5.

72. Afridi SK, Matharu MS, Lee L, et al. A PET study exploring the laterality of brainstem activation in migraine using glyceryltrinitrate. Brain. 2005;128: 932-9.

73. Schwedt TJ, Chong CD. Functional imaging and migraine: new connections? Curr Opin Neurol. 2015;28:265-70.

74. Martín H, del Río MS, de Silanes CL, Álvarez-Linera J, Hernández JA, Pareja JA. Photoreactivity of the occipital cortex measured by functional magnetic resonance imaging-blood oxygenation level dependent in migraine patients and healthy 
volunteers: pathophysiological implications. Headache. 2011;51:1520-8.

75. Vincent M, Pedra E, Mourão-Miranda J, Bramati IE, Henrique AR, Moll J. Enhanced interictal responsiveness of the migraineous visual cortex to incongruent bar stimulation: a functional MRI visual activation study. Cephalalgia. 2003;23:860-8.

76. Rocca Maria A, Colombo B, Pagani E, et al. Evidence for cortical functional changes in patients with migraine and white matter abnormalities on conventional and diffusion tensor magnetic resonance imaging. Stroke. 2003;34:665-70.

77. Antal A, Polania R, Saller K, et al. Differential activation of the middle-temporal complex to visual stimulation in migraineurs. Cephalalgia. 2011;31: $338-45$.

78. Moulton EA, Becerra L, Maleki N, et al. Painful heat reveals hyperexcitability of the temporal pole in interictal and ictal migraine states. Cereb Cortex. 2011;21:435-48.

79. Russo A, Tessitore A, Esposito F, et al. Pain processing in patients with migraine: an event-related fMRI study during trigeminal nociceptive stimulation. J Neurol. 2012;259:1903-12.

80. Tessitore A, Russo A, Esposito F, et al. Interictal cortical reorganization in episodic migraine without aura: an event-related fMRI study during parametric trigeminal nociceptive stimulation. Neurol Sci. 2011;32(Suppl 1):S165-167.

81. Mainero C, Boshyan J, Hadjikhani N. Altered functional magnetic resonance imaging resting-state connectivity in periaqueductal gray networks in migraine. Ann Neurol. 2011;70:838-45.

82. Niddam DM, Lai KL, Fuh JL, Chuang CY, Chen WT, Wang SJ. Reduced functional connectivity between salience and visual networks in migraine with aura. Cephalalgia. 2016;36:53-66.

83. Tedeschi G, Russo A, Conte F, et al. Increased interictal visual network connectivity in patients with migraine with aura. Cephalalgia. 2016;36: 139-47.

84. Hougaard A, Amin FM, Magon S, Sprenger T, Rostrup E, Ashina M. No abnormalities of intrinsic brain connectivity in the interictal phase of migraine with aura. Eur J Neurol. 2015;22:702-e746.

85. Coppola G, Di Renzo A, Tinelli E, et al. Thalamocortical network activity during spontaneous migraine attacks. Neurology. 2016;87:2154-60.

86. Coppola G, Di Renzo A, Tinelli E, et al. Resting state connectivity between default mode network and insula encodes acute migraine headache. Cephalalgia. 2018;38:846-54.

87. Coppola G, Di Renzo A, Petolicchio B, et al. Aberrant interactions of cortical networks in chronic migraine: a resting-state fMRI study. Neurology. 2019;92:e2550-8.

88. Androulakis XM, Rorden C, Peterlin BL, Krebs K. Modulation of salience network intranetwork resting state functional connectivity in women with chronic migraine. Cephalalgia. 2018;38:1731-41.

89. Younis S, Hougaard A, Vestergaard MB, Larsson HBW, Ashina M. Migraine and magnetic resonance spectroscopy: a systematic review. Curr Opin Neurol. 2017;30:246-62.

90. Scheau C, Preda EM, Popa GA, Ghergus AE, Capsa RA, Lupescu IG. Magnetic resonance spectroscopya non-invasive method in evaluating focal and diffuse central nervous system disease. J Med Life. 2012;5:423-7.

91. Welch KM, Ramadan NM. Mitochondria, magnesium and migraine. J NeurolSci. 1995;134:9-14.

92. Lodi R, Montagna P, Soriani S, et al. Deficit of brain and skeletal muscle bioenergetics and low brain magnesium in juvenile migraine: an in vivo 31P magnetic resonance spectroscopy interictal study. Pediatr Res. 1997;42:866-71.

93. Barbiroli B, Montagna P, Cortelli P, et al. Abnormal brain and muscle energy metabolism shown by $31 \mathrm{P}$ magnetic resonance spectroscopy in patients affected by migraine with aura. Neurology. 1992;42: 1209-14.

94. Welch KM, Levine SR, D'Andrea G, Schultz LR, Helpern JA. Preliminary observations on brain energy metabolism in migraine studied by in vivo phosphorus 31 NMR spectroscopy. Neurology. 1989;39:538-41.

95. Schulz UG, Blamire AM, Corkill RG, Davies P, Styles $\mathrm{P}$, Rothwell PM. Association between cortical metabolite levels and clinical manifestations of migrainous aura: an MR-spectroscopy study. Brain. 2007; 130:3102-10.

96. Montagna P, Cortelli P, Monari L, et al. 31P-magnetic resonance spectroscopy in migraine without aura. Neurology. 1994;44:666-9.

97. Reyngoudt H, Paemeleire K, Descamps B, De Deene $\mathrm{Y}$, Achten E. 31P-MRS demonstrates a reduction in high-energy phosphates in the occipital lobe of migraine without aura patients. Cephalalgia. 2011;31:1243-53. 
98. Ramadan NM, Halvorson H, Vande-Linde A, Levine SR, Helpern JA, Welch KM. Low brain magnesium in migraine. Headache. 1989;29:590-3.

99. Lodi R, Iotti S, Cortelli P, et al. Deficient energy metabolism is associated with low free magnesium in the brains of patients with migraine and cluster headache. Brain Res Bull. 2001;54:437-41.

100. Boska MD, Welch KM, Barker PB, Nelson JA, Schultz L. Contrasts in cortical magnesium, phospholipid and energy metabolism between migraine syndromes. Headache J Head Face Pain. 2003;43: $425-425$.

101. Bridge H, Stagg CJ, Near J, Lau CI, Zisner A, Cader MZ. Altered neurochemical coupling in the occipital cortex in migraine with visual aura. Cephalalgia. 2015;35:1025-30.

102. Siniatchkin M, Sendacki M, Moeller F, et al. Abnormal changes of synaptic excitability in migraine with aura. Cereb Cortex. 2012;22: 2207-16.

103. de la Aleja JG, Ramos A, Mato-Abad V, et al. Higher glutamate to glutamine ratios in occipital regions in women with migraine during the interictal state. Headache J Head Face Pain. 2013;53:365-75.

104. Bigal ME, Hetherington $\mathrm{H}$, Pan J, et al. Occipital levels of GABA are related to severe headaches in migraine. Neurology. 2008;70:2078-80.

105. Mohamed RE, Aboelsafa AA, Al-Malt AM. Interictal alterations of thalamic metabolic concentration ratios in migraine without aura detected by proton magnetic resonance spectroscopy. Egypt J Radiol Nucl Med. 2013;44:859-70.

106. Gu T, Ma XX, Xu YH, Xiu JJ, Li CF. Metabolite concentration ratios in thalami of patients with migraine and trigeminal neuralgia measured with 1H-MRS. Neurol Res. 2008;30:229-33.

107. Sarchielli P, Tarducci R, Presciutti O, et al. Functional $1 \mathrm{H}-\mathrm{MRS}$ findings in migraine patients with and without aura assessed interictally. Neuroimage. $2005 ; 24: 1025-31$.

108. Tkác I, Oz G, Adriany G, Uğurbil K, Gruetter R. In vivo $1 \mathrm{H}$ NMR spectroscopy of the human brain at high magnetic fields: metabolite quantification at 4T vs. 7T. Magn Reson Med. 2009;62:868-79.

109. Portnow LH, Vaillancourt DE, Okun MS. The history of cerebral PET scanning: from physiology to cutting-edge technology. Neurology. 2013;80: 952-6.

110. Deen M, Hansen HD, Hougaard A, et al. Low 5 -HT1B receptor binding in the migraine brain: a PET study. Cephalalgia. 2017;38:519-27.

111. Albrecht DS, Mainero C, Ichijo E, et al. Imaging of neuroinflammation in migraine with aura: a [(11)C]PBR28 PET/MRI study. Neurology. 2019;92: e2038-50.

112. Hadjikhani N, Vincent M. Neuroimaging clues of migraine aura. J Headache Pain. 2019;20:32.

113. Hadjikhani N, Albrecht DS, Mainero C, et al. Extraaxial inflammatory signal in parameninges in migraine with visual aura. Ann Neurol. 2020;87: 939-49.

114. Denuelle M, Fabre N, Payoux P, Chollet F, Geraud G. Posterior cerebral hypoperfusion in migraine without aura. Cephalalgia. 2008;28:856-62.

115. Tfelt-Hansen P, Olesen J. Posterior hypoperfusion in migraine without aura? Cephalalgia. 2010;30:1018 (author reply 1018-1020).

116. Géraud G, Denuelle M, Fabre N, Payoux P, Chollet F. Positron emission tomographic studies of migraine. Rev Neurol (Paris). 2005;161:666-70.

117. Fumal A, Laureys S, Di Clemente L, et al. Orbitofrontal cortex involvement in chronic analgesicoveruse headache evolving from episodic migraine. Brain. 2006;129:543-50. 